\title{
Efecto de la temperatura, basicidad, ciclo térmico y potencial oxidante en la formación de fases minerales en briquetas de mineral de hierro $^{(\bullet)}$
}

\author{
E. Bedolla ${ }^{(*)}$, E.A. Aguilar ${ }^{(*)}$ y C.A. León ${ }^{(*)}$ \\ Resumen Se estudió el efecto de las condiciones de sinterización tales como temperatura, basicidad, ciclo térmi- \\ co y potencial oxidante en la formación de fases minerales en briquetas de mineral de hierro magnetí- \\ tico. Se encontró que un alto potencial de oxígeno (aire) favorece la reacción de oxidación de magneti- \\ ta a hematites y como consecuencia la formación de calcioferrita. La calcioferrita se incrementó con el \\ aumento de la basicidad y disminuyó con el incremento de la temperatura. Para un potencial de oxíge- \\ no medio $\left(\mathrm{pO}_{2}=5 \times 10^{-3} \mathrm{~atm}\right.$.), el aglomerado estuvo compuesto en su mayor parte por magnetita con \\ pequeñas cantidades de hematites reoxidada y cantidades insignificantes de calcioferrita. Se observó \\ que la magnetita se incrementa y la hematites disminuye con el aumento de la basicidad. Las fases \\ minerales presentes en briquetas sinterizadas en la mezcla gaseosa de $1 \% \mathrm{CO}, 24 \% \mathrm{CO}_{2}$ y $75 \% \mathrm{~N}_{2}$, \\ incluyen la magnetita y fases altamente oxidadas como hematites y calcioferrita.
}

Palabras clave: Aglomeración. Fases minerales. Potencial oxidante. Mineral de hierro. Sinterización.

\section{Effect of temperature, basicity, firing cycle and oxygen potential on mineral phases in iron ore briquettes}

\begin{abstract}
In the present work, the effect of the firing conditions such as temperature, basicity, firing cycle and oxygen potential, on the formation of mineral phases in magnetitic iron ore briquettes was studied. It was found that a high oxygen potential (air) favors the oxidation reaction of magnetite to hematite and as a consequence calcium ferrite formation. The calcium ferrite increased with the increase of the basicity and decreased with the increase of the temperature. At a medium partial pressure of oxygen $\left(\mathrm{pO}_{2}=5 \times 10^{-3} \mathrm{~atm}\right.$.), the agglomerate was composed in the majority of magnetite with small amounts of reoxidized hematite and negligible amounts of calcium ferrite. It could be noticed that magnetite increased and hematite decreased with increase of basicity. The mineral phases present in the briquettes fired at the gas mixture composed by $1 \% \mathrm{CO}, 24 \% \mathrm{CO}_{2}$ and $75 \% \mathrm{~N}_{2}$ and then cooled in air, include magnetite and highly oxidized phases such as hematite and calcium ferrite.
\end{abstract}

Keywords: Agglomeration. Mineral phases. Oxygen potential. Iron ore. Sintering.

\section{INTRODUCCIÓN}

Numerosos investigadores han estudiado el efecto de las condiciones prevalecientes durante el quemado de aglomerados de mineral de hierro sobre la microestructura final y sus propiedades físicas y mecánicas, debido a que éstas se hallan grandemente influidas por el tipo, características, cantidad y

(•) Trabajo recibido el día 27 de agosto de 1996.

(*) Instituto de Investigaciones Metalúrgicas. Universidad Michoacana de San Nicolás de Hidalgo. Apdo. Postal 52-B. Morelia, Mich. (México). distribución de las fases minerales presentes en el aglomerado.

Primeros estudios (1-3) revelaron que los dicalciosilicatos y las escorias vítreas constituyen el principal agente de unión en los aglomerados, ya que están presentes como una matriz continua que encierra los granos de óxidos de hierro y proporcionan buena resistencia a los compactos. Sin embargo, en grandes proporciones pueden ser perjudiciales. La escoria vítrea tiene un alto grado de tensión y fatiga atribuido a una diferencia en el coeficiente térmico de expansión-contracción entre la fase vítrea y la fase cristalina que encierra. Por otro 
lado, cuando aparecen como un producto de la desvitrificación del vidrio, los dicalciosilicatos son perjudiciales para la resistencia del sínter, ya que a 697 ${ }^{\circ} \mathrm{C}$ el dicalciosilicato sufre una transformación alotrópica de $\alpha-2 \mathrm{CaO} \cdot \mathrm{SiO}_{2}$ a $\gamma-2 \mathrm{CaO} \cdot \mathrm{SiO}_{2}$, acompañada por un cambio de volumen que trae como consecuencia la desintegración del sínter.

Recientemente, se investigó el efecto de la adición de serpentina sobre la resistencia mecánica y la reducibilidad de briquetas de mineral de hierro (4 y 5). De los resultados experimentales se concluyó que la presencia de ciertas fases minerales, como la olivina y las ferritas de calcio, influyen grandemente en la resistencia mecánica y en la reducibilidad y, por el contrario, fases como la escoria vítrea y las magnesioferritas disminuyen estas propiedades.

Friel y Erickson, Jr. (6 y 7), estudiaron el efecto de la dolomita en las propiedades físicas, mecánicas y de reducción de pelets de magnetita. Consideran que existen tres factores que desempeñan un papel importante en los pelets con adiciones de fundente: el contenido de $\mathrm{MgO}$, la relación $\mathrm{CaO} / \mathrm{SiO}_{2}$ y las condiciones de sinterización.

Si el nivel de $\mathrm{MgO}$ es muy bajo, los pelets no contienen suficiente fundente, y se observa sólo un ligero mejoramiento de las propiedades en comparación con las de los pelets ácidos; por el contrario, si el nivel de $\mathrm{MgO}$ es muy alto, el fundente sin reaccionar debilita la estructura. La relación $\mathrm{CaO} / \mathrm{SiO}_{2}$ controla la cantidad de escoria producida durante la sinterización y, por lo tanto, la cantidad de $\mathrm{MgO}$ que puede ser transportado a los óxidos de hierro para formar magnesioferrita. Si la relación $\mathrm{CaO} / \mathrm{SiO}_{2}$ es muy baja, la escoria es viscosa y solamente se forma una pequeña cantidad de magnesioferrita. Si la relación es muy alta, se encuentran presentes cal libre o calcioferrita, dando como resultado una degradación a baja temperatura. Por último, dichos autores señalan que la temperatura máxima de sinterización debería ser optimizada. Una temperatura baja produce un debilitamiento, aunque los pelets producidos sean altamente reducibles, y una temperatura muy alta produce un pelet denso con baja reducibilidad.

Panigrahy y cols. (3) señalaron que, en general, el incremento de la relación $\mathrm{MgO} / \mathrm{CaO}$ contribuye a disminuir la resistencia en frío del sínter, pero para sínteres de baja basicidad, pequeñas adiciones tienen un efecto beneficioso. Para sínteres ricos en $\mathrm{CaO}$, la presencia de calcioferritas es la principal fase que contribuye a la resistencia, mientras que para sínteres ricos en $\mathrm{MgO}$, la precipitación de olivinas en la matriz vítrea contribuye a la más alta resistencia. La presencia de grandes charcos de escoria y/o la precipitación de dicalciosilicatos parece tener un efecto perjudicial sobre la resistencia de los sínteres.

Hsieh y cols. (8 y 9) realizaron un amplio estudio sobre el efecto del potencial de oxígeno en la formación de fases minerales en aglomerados de mineral de hierro. Encontraron que, durante la etapa de calentamiento de la sinterización industrial, la calcioferrita acicular se genera inicialmente por la reacción de la hematites y el fundente (que contiene $\mathrm{CaO}, \mathrm{SiO}_{2}$ y $\mathrm{Al}_{2} \mathrm{O}_{3}$ ). Al aumentar la temperatura, aumenta la cantidad de calcioferrita acicular así como el tamaño de sus cristales y posteriormente se disocia en magnetita y en silicato líquido.

Señalaron, además, que un mayor potencial oxidante favorece la formación de calcioferrita acicular en la etapa de calentamiento, y que ésta es producida por un mineral hematítico a menor potencial oxidante. La cantidad de hematites producida por la oxidación de la magnetita en los especímenes de mineral magnetítico, también se incrementa con el potencial de oxígeno.

Igualmente, encontraron que, durante la etapa de enfriamiento, la generación de la calcioferrita (de la magnetita y silicato líquido) se produce a una presión parcial media de oxígeno (alrededor de $1 \times 10^{-2}$ atm.). La formación de hematites reoxidada ocurre a ún potencial de oxígeno elevado y la magnetita (formada en la etapa de calentamiento) se conserva en una atmósfera de potencial de oxígeno bajo.

De todo lo anterior, se concluye que, para un completo establecimiento de los procesos de formación mineral de los aglomerados, es necesario estudiarlos como una función de las diversas condiciones, tales como composición química, composición mineral, temperatura y período de calentamiento, tamaño de partícula, atmósfera, etc.

Para investigar el efecto de las condiciones de sinterización en la formación de fases minerales, se quemaron briquetas de mineral de hierro magnetítico; además, la proporción del volumen de cada una de las fases minerales presentes se cuantificó mediante el método de recuento de puntos.

\section{PROCEDIMIENTO EXPERIMENTAL}

\subsection{Materiales}

Para la realización de este estudio se utilizó mineral magnetítico de las minas de Las Truchas, S.A., de Michoacán (México), además de óxido de calcio de grado reactivo con el que se ajustó la relación de basicidad binaria $\left(\mathrm{CaO} / \mathrm{SiO}_{2}\right)$ de las mezclas. El mineral de hierro utilizado es de granulometría fina, aproximadamente el 79,5\% < $44 \mu \mathrm{m}$. La composición química tanto del mineral como del óxido de calcio se muestra en la tabla I.

\subsection{Preparación de las muestras}

Se fabricaron probetas cilíndricas de aproximadamente $8 \mathrm{~mm}$ de diámetro y $4 \mathrm{~mm}$ de espesor, de 
TABLA I.- Composición química de los materiales y de las mezclas preparadas

TABLE I.- Chemical composition of raw materials and mixtures prepared

\begin{tabular}{|c|c|c|c|c|c|c|c|}
\hline Material & $\mathrm{Fe}_{\mathrm{T}}$ & $\mathrm{Fe}^{2+}$ & $\mathrm{SiO}_{2}$ & $\mathrm{CaO}$ & $\mathrm{MgO}$ & $\mathrm{Al}_{2} \mathrm{O}_{3}$ & $\mathrm{~S}$ \\
\hline $\mathrm{Fe}_{3} \mathrm{O}_{4}$ & 65,59 & 25,512 & 3,21 & 1,47 & 0,380 & 0,750 & 0,0108 \\
$\mathrm{Cal}$ & - & - & - & 99,00 & - & - & - \\
\hline $\mathrm{CaO} / \mathrm{SiO}_{2}$ & $\mathrm{Fe}_{\mathrm{T}}$ & $\mathrm{Fe}^{+2}$ & $\mathrm{SiO}_{2}$ & $\mathrm{CaO}$ & $\mathrm{MgO}$ & $\mathrm{Al}_{2} \mathrm{O}_{3}$ & $\mathrm{~S}$ \\
\hline 1,0 & 64,47 & - & 3,16 & 3,16 & 0,374 & 0,737 & 0,0106 \\
1,6 & 63,27 & - & 3,10 & 4,95 & 0,367 & 0,723 & 0,0104 \\
2,0 & 62,50 & - & 3,06 & 6,12 & 0,362 & 0,715 & 0,0103 \\
\hline
\end{tabular}

$\mathrm{Fe}_{\mathrm{T}}=$ Contenido de Fe total

mezclas de mineral de hierro magnetítico y cal con basicidades de $1,1,6$ y 2 , al prensar $0,8 \mathrm{~g}$ de la mezcla de material con $8 \%$ de agua como aglutinante en un cubo de acero a una presión de 200 $\mathrm{kg} / \mathrm{cm}^{2}$. La composición química de las mezclas resultantes se muestra también en la tabla I.

\subsection{Quemado de las muestras}

Antes de someterlas al proceso de sinterización, las probetas se secaron a $100{ }^{\circ} \mathrm{C}$ durante $3 \mathrm{~h}$. Los ensayos de sinterización se efectuaron en un horno tubular de alta temperatura calentado por resistencias eléctricas, que consta de un tubo de alúmina de $3 \mathrm{~cm}$ de diámetro interior y $55 \mathrm{~cm}$ de longitud. La temperatura de la muestra se controló por medio de un termopar de Pt/Pt-13\% Rh colocado exactamente debajo del contenedor de la muestra.

Las probetas se calentaron desde la temperatura ambiente hasta $1.000{ }^{\circ} \mathrm{C}$ a una velocidad de 70 ${ }^{\circ} \mathrm{C} / \mathrm{min}$. A partir de esta temperatura, a una velocidad de calentamiento de $60^{\circ} \mathrm{C} / \mathrm{min}$, se alcanzaron las temperaturas máximas de 1.250 y $1.300{ }^{\circ} \mathrm{C}$, en las que las probetas se mantuvieron durante $10 \mathrm{~min}$ y posteriormente se enfriaron a velocidades de 20 , 40 y $60{ }^{\circ} \mathrm{C} / \mathrm{min}$. La representación gráfica del tiempo en función de la temperatura de los ensayos de sinterización se muestra en la figura 1.

Los ensayos de sinterización se realizaron en aire $\left(\mathrm{pO}_{2}=0,21 \mathrm{~atm}\right.$. $)$ y en una atmósfera con 99,5 $\% \mathrm{~N}_{2}$ y $0,5 \% \mathrm{O}_{2}\left(\mathrm{pO}_{2}=5 \times 10^{-3}\right.$ atm. $)$, empleándose las mismas mezclas en el enfriamiento. Durante el calentamiento, se utilizó una tercera mezcla gaseosa compuesta por $24 \% \mathrm{CO}_{2}, 1 \% \mathrm{CO}$ y $75 \% \mathrm{~N}_{2}$ y a continuación se enfrió al aire. De acuerdo con algunos autores (8-10), esta última mezcla gaseosa se considera como estándar en los ensayos para simular el proceso de sinterización industrial. Para calcular el potencial de oxígeno de la mezcla gaseosa que contiene $\mathrm{CO}$ en la sinterización, se utiliza la

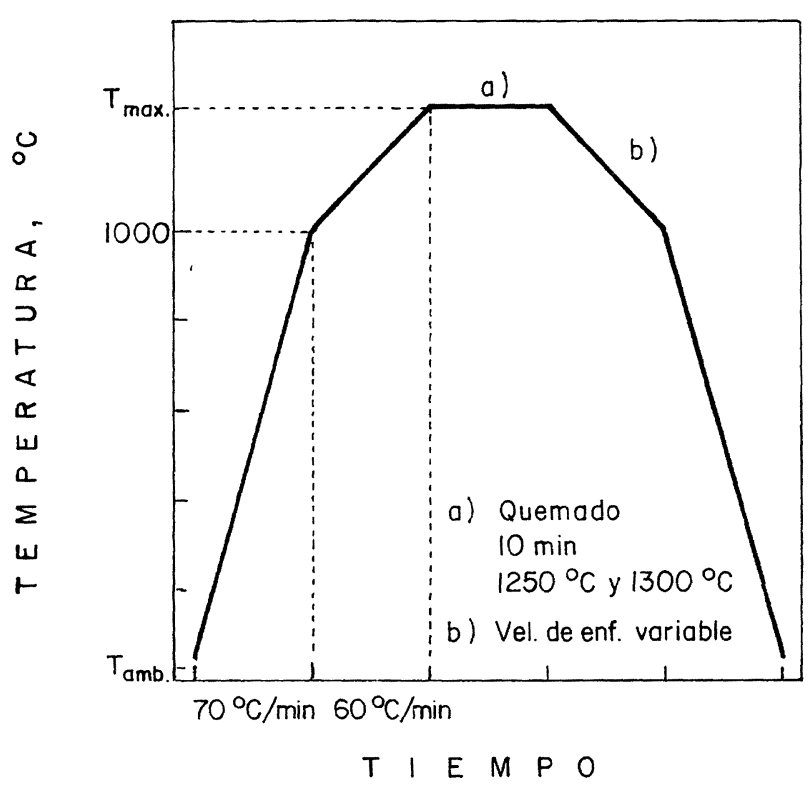

FIG. 1.- Ciclo térmico experimental.

FIG. 1.- Experimental firing cycle.

siguiente reacción química y la ecuación de energía libre estándar:

$$
\begin{gathered}
\mathrm{CO}+{ }^{1 / 2} \mathrm{O}_{2} \leftrightarrow \mathrm{CO}_{2} \\
\Delta G=-67500+20,75 T
\end{gathered}
$$

donde, $\Delta G=$ Energía libre estándar, $\mathrm{cal} / \mathrm{mol}$.

$T=$ Temperatura máxima del gas, $\mathrm{K}$.

El flujo total de cada una de las mezclas fue de $200 \mathrm{~cm}^{3} / \mathrm{min}$.

\subsection{Identificación y cuantificación de las fases minerales}

Con el propósito de identificar tanto cualitativa como cuantitativamente las fases de escoria y 
ferrita cálcica, además de observar en detalle la morfología de las fases minerales presentes, se llevó a cabo una serie de estudios microscópicos de probetas previamente pulidas y fracturadas. Dichos estudios se realizaron en un microscopio electrónico de barrido, MEB, marca JEOL JSM-6400 con microanalizador de dispersión de energías incorporado.

Después de la sinterización, las probetas se desbastaron utilizando papel de carburo de silicio, con etanol como lubricante $y$, finalmente, se pulieron con pasta de diamante de $1 \mu \mathrm{m}$. Durante el proceso completo de pulido, las probetas sinterizadas no fueron expuestas al agua.

Las probetas se examinaron utilizando un microscopio óptico de luz reflejada, y la proporción del volumen de las fases en cada probeta se estimó utilizando el método del recuento de puntos (9-11). El método se fundamenta en la fracción del número total de puntos analizados que caen en la superficie correspondiente a una microestructura específica seleccionada, analizándose un total de 500 puntos. Las proporciones de volumen de cada fase se calcularon de la siguiente forma:

$$
\begin{gathered}
\% \text { Fase } \alpha=\frac{N P \alpha}{N P T} \times 100 \\
\% \text { Porosidad }=\frac{N P \text { poros }}{N P \text { poros }+N P T} \times 100
\end{gathered}
$$

donde, $\quad N P \alpha=$ Número de puntos de la fase $\alpha$. $N P T=$ Número de puntos totales de todas las fases.

$N P$ poros $=$ Número de puntos de los poros.

\section{RESULTADOS Y DISCUSIÓN}

\subsection{Efecto del potencial oxidante en la formación de fases minerales}

Las micrografías ópticas de muestras pulidas y sinterizadas en los tres potenciales oxidantes, así como las proporciones de las fases minerales resultantes del recuento de puntos, se muestran en las figuras 2 y 3 . De la figura 2 se observa que la calcioferrita se generó a partir del mineral magnetítico cuando la sinterización se llevó a cabo en aire $\left(\mathrm{pO}_{2}\right.$ $=0,21$ atm.); sin embargo, en la mezcla gaseosa de $\mathrm{N}_{2}=99,5 \%$ y $\mathrm{O}_{2}=0,5 \%\left(\mathrm{pO}_{2}=5 \times 10^{-3} \mathrm{~atm}\right.$. $)$ se formaron cantidades insignificantes de calcioferrita. De lo anterior, se deduce que un elevado potencial de oxígeno favorece la formación de calcioferrita en la etapa de calentamiento. Se puede observar que cuando el potencial de oxígeno se incrementa, la

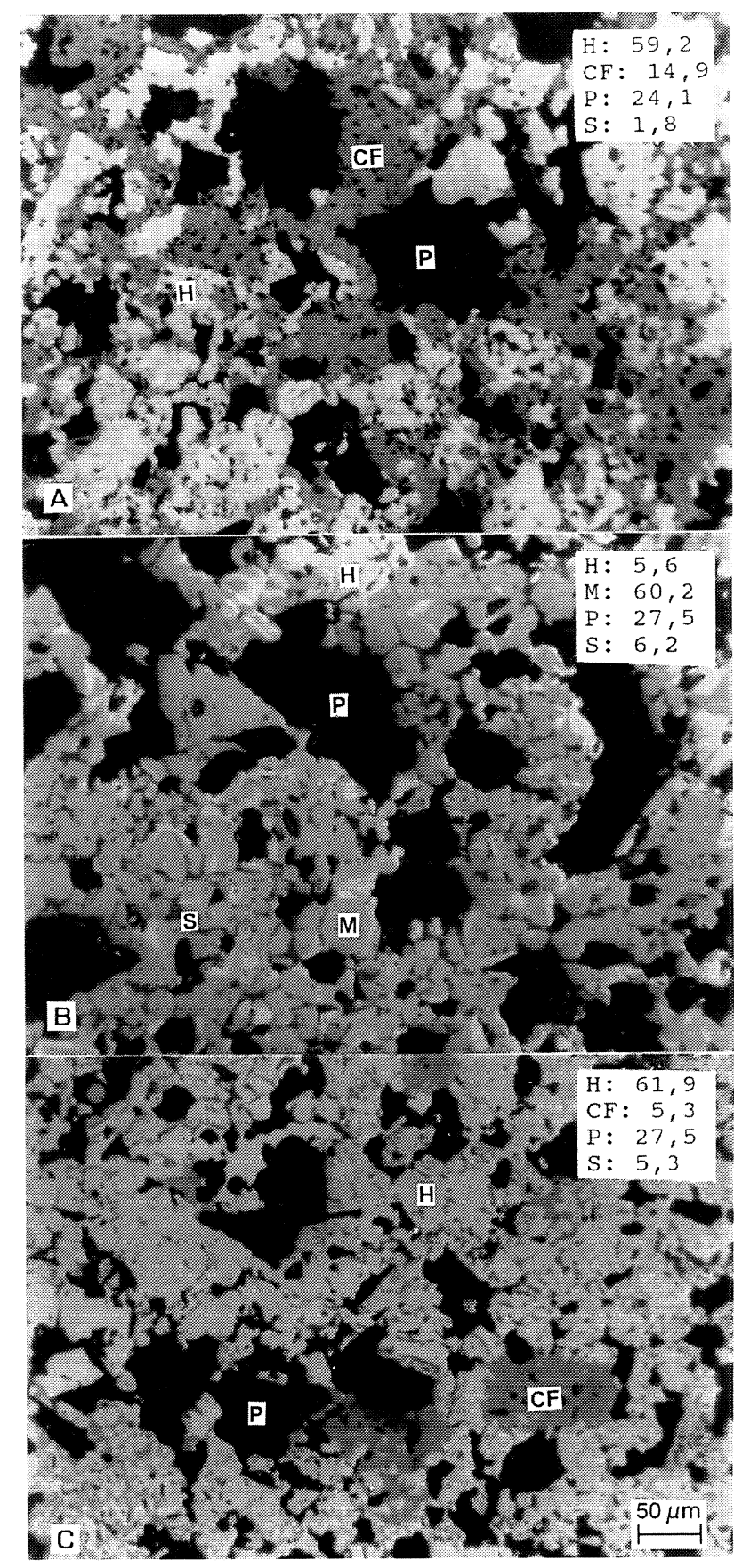

FIG. 2.- Micrografías de muestras pulidas y sinterizadas a $1.250{ }^{\circ} \mathrm{C}$ en: a) Aire, b) $99,5 \% \mathrm{~N}_{2}-0,5 \%$ $\mathrm{O}_{2}$, c) $1 \% \mathrm{CO}-24 \% \mathrm{CO}_{2}-75 \% \mathrm{~N}_{2}$-Aire. $\mathrm{H}$ : hematites, M: magnetita, CF: calcioferrita, S: escoria, P: poro.

FIG. 2.- Photomicrographs of polished samples fired at $1,250{ }^{\circ} \mathrm{C}$ in: a) Air, b) $99.5 \% \mathrm{~N}_{2}-0.5 \% \mathrm{O}_{2}$, c) $1 \% \mathrm{CO}-24 \% \mathrm{CO}_{2}-75 \% \mathrm{~N}_{2}$-Air. H: hematite, $M$ : magnetite, $C F$ : calcium ferrite, $S$ : slag, $P$ : pore.

magnetita se oxida a hematites y la porosidad disminuye debido al cambio de volumen que origina la transformación de la estructura cristalina cúbica de la magnetita a la estructura hexagonal de la hematites. La oxidación de la magnetita comienza 


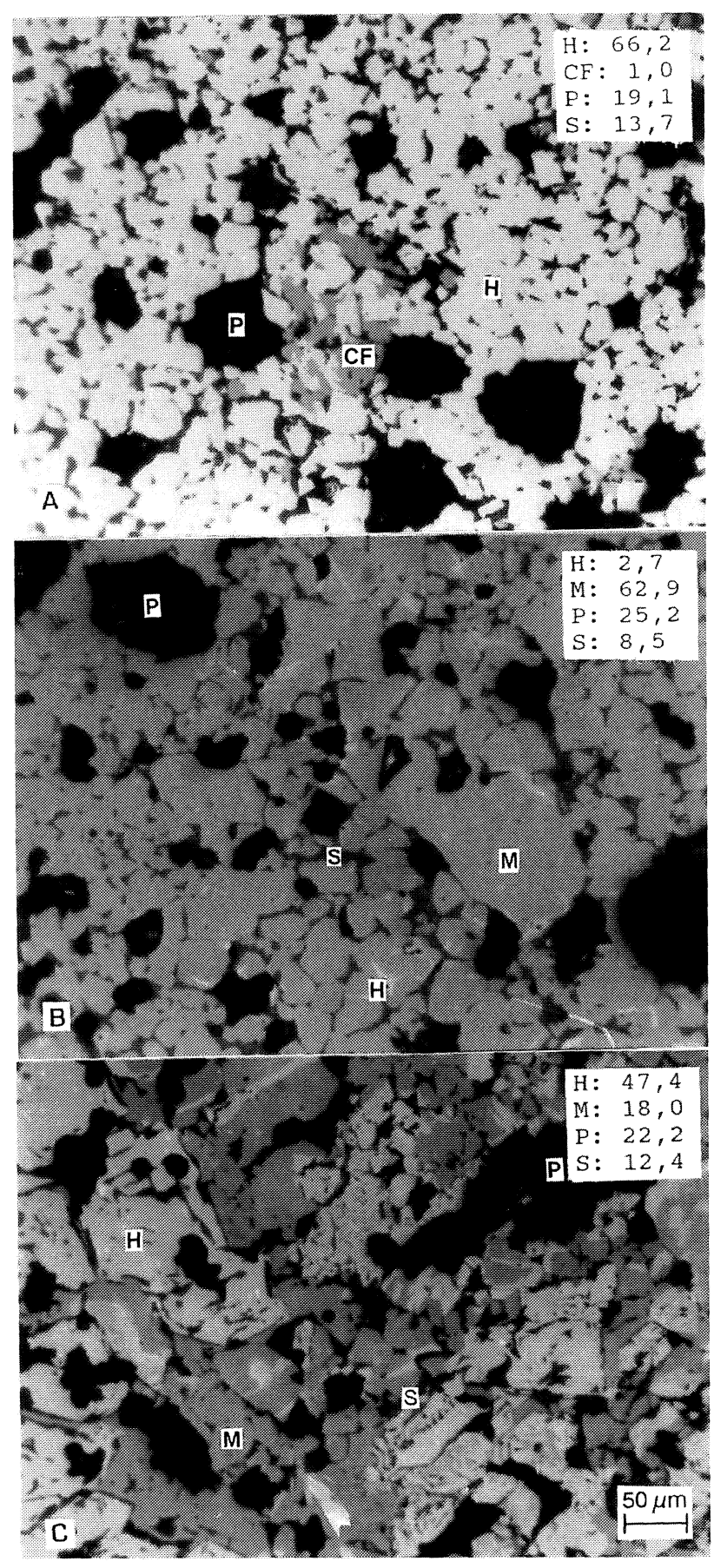

FIG. 3.- Micrografías de muestras pulidas y sinterizadas a $1.300{ }^{\circ} \mathrm{C}$ en: a) Aire, b) $99,5 \% \mathrm{~N}_{2}-0,5 \%$ $\mathrm{O}_{2}$, c) $1 \% \mathrm{CO}-24 \% \mathrm{CO}_{2}-75 \% \mathrm{~N}_{2}$-Aire. $\mathrm{H}$ : hematites, M: magnetita, CF: calcioferrita, S: escoria, P: poro.

FIG. 3.- Photomicrographs of polished samples fired at $1,300{ }^{\circ} \mathrm{C}$ in: a) Air, b) $99.5 \% \mathrm{~N}_{2}-0.5 \% \mathrm{O}_{2}$, c) $1 \% \mathrm{CO}-24 \% \mathrm{CO}_{2}-75 \% \mathrm{~N}_{2}$-Air. H: hematite, M: magnetite, CF: calcium ferrite, S: slag, P: pore.

alrededor de $300-600{ }^{\circ} \mathrm{C}$ y termina a $1.100{ }^{\circ} \mathrm{C}$, iniciándose en la superficie de los cristales y granos (12).
Del mismo modo, se puede observar un incremento de la cantidad de hematites con un elevado potencial oxidante. Por esta razón, durante la etapa de calentamiento la hematites constituye una fase intermedia para la formación de calcioferrita cuando se parte de mezclas de mineral magnetítico. En una atmósfera de elevado potencial de oxígeno, la magnetita se oxida inicialmente a hematites y la calcioferrita se genera a partir de la reacción de la hematites y el fundente.

Cuando la sinterización se realizó a un potencial medio de oxígeno $\left(\mathrm{pO}_{2}=5 \times 10^{-3} \mathrm{~atm}\right.$. $)$, la mayoría de la magnetita que compone el mineral original se conservó al final de la sinterización y del enfriamiento, y sólo se observaron pequeñas cantidades de hematites reoxidada, alrededor del $6 \%$.

Las figuras 2c) y 3c) muestran las fases minerales presentes en las briquetas sinterizadas en la mezcla gaseosa compuesta por $1 \% \mathrm{CO}, 24 \% \mathrm{CO}_{2}$ y $75 \% \mathrm{~N}_{2}$, y que son las mismas que las formadas en las dos atmósferas mencionadas anteriormente. Debido a que el calentamiento se realizó en atmósfera reductora, al final de la sinterización y del enfriamiento se conserva una cantidad considerable de la magnetita que compone el mineral original. También se presentan fases muy oxidadas, como hematites y calcioferrita, que se forman durante el enfriamiento al aire, la primera, por oxidación de la magnetita y la segunda como producto de la reacción de la magnetita, silicato líquido y oxígeno del aire.

En la tabla II se resumen las fases minerales predominantes en los aglomerados en función del potencial oxidante y de la temperatura de sinterización. Dichas fases se forman en diferente proporción con cada uno de los valores de los índices de basicidad estudiados.

TABLA II.- Fases minerales predominantes en los aglomerados en función del potencial oxidante y de la temperatura de sinterización

TABLE II.- Predominant mineral phases in the agglomerates as a function of oxygen potential and firing temperature

\begin{tabular}{|c|ccc|}
\hline $\begin{array}{c}\text { Temp. de } \\
\text { sint., }{ }^{\circ} \mathrm{C}\end{array}$ & $\begin{array}{c}\text { Aire } \\
\left(\mathrm{PO}_{2}=0,21\right. \\
\text { atm. })\end{array}$ & $\begin{array}{c}0,5 \% \mathrm{O}_{2} \mathrm{y} \\
99,5 \% \mathrm{~N}_{2} \\
\left(\mathrm{PO}_{2}=5 \times 10^{-3}\right. \\
\text { atm. })\end{array}$ & $\begin{array}{c}1 \% \mathrm{CO}, 24 \% \\
\mathrm{CO}_{2}, \mathrm{y} 75 \% \\
\mathrm{~N}_{2} \text {-Aire }\end{array}$ \\
\hline 1.250 & $\mathrm{H}, \mathrm{CF}, \mathrm{S}, \mathrm{P}$ & $\mathrm{M}, \mathrm{H}, \mathrm{S}, \mathrm{P}$ & $\mathrm{H}, \mathrm{CF}, \mathrm{S}, \mathrm{P}$ \\
1.300 & $\mathrm{H}, \mathrm{CF}, \mathrm{S}, \mathrm{P}$ & $\mathrm{M}, \mathrm{H}, \mathrm{S}, \mathrm{P}$ & $\mathrm{H}, \mathrm{M}, \mathrm{S}, \mathrm{P}$ \\
\hline
\end{tabular}

$\mathrm{H}$ : hematites, CF: calcioferritas, M: magnetita, S: escoria, P: poro 


\subsection{Efecto de la basicidad y de la temperatura en la formación de fases minerales}

\subsubsection{Briquetas sinterizadas en aire}

Los resultados para las proporciones de las fases minerales se muestran en la figura 4 como una función de la basicidad para diferentes temperaturas, y las micrografías de estas mismas fases se muestran en la figura 5. En las figuras 4 y 5 , se puede observar que para las probetas sinterizadas al aire, la cantidad de calcioferrita se incrementa y la cantidad de hematites disminuye con el incremento de la basicidad. Estos hechos se atribuyen a la reacción en el estado sólido que ocurre durante el calentamiento (alrededor de $950-1.100{ }^{\circ} \mathrm{C}$ ) para generar calcioferrita. Dicha relación, en la cual se consumen hematites y cal, se ve favorecida por el incremento de la basicidad (relación $\mathrm{CaO} / \mathrm{SiO}_{2}$ ).

En general, la porosidad disminuye en los compactos con el aumento de la basicidad, es decir, con el contenido de $\mathrm{CaO}$ en la muestra. Lo anterior ha sido observado por otros investigadores (13) que señalan que a temperaturas de $1.250-1.350{ }^{\circ} \mathrm{C}$, la velocidad de disminución de la porosidad se acelera acusadamente con el incremento del contenido de $\mathrm{CaO}$. Esta disminución del volumen de los poros parece deberse principalmente a la eliminación, por mecanismos de sinterización, de los poros más finos en las estructuras, ya que la adición de cantidades relativamente pequeñas de $\mathrm{CaO}$ a los compactos acelera fuertemente las reacciones de sinterización cuando se forma una fase líquida.

La escoria, cuya formación se produce rápidamente y es mucho más fácilmente perceptible con un aumento del contenido de $\mathrm{CaO}$, forma una matriz casi continua alrededor de las partículas de óxidos y es en este estado en el que se desarrollan los poros cerrados debido al incremento del tamaño promedio de las partículas o al crecimiento de grano, acompañado por una disminución de la porosidad del compacto por eliminación de poros finos.

A $1.300{ }^{\circ} \mathrm{C}$, la proporción de escoria en las probetas aumenta con la basicidad. Se sabe que el principal efecto de los aditivos es facilitar la formación de escoria, especialmente por la disolución de cuarzo. Un incremento de la cantidad de $\mathrm{CaO}$ en el compacto de mineral de hierro da como resultado la formación de una escoria líquida de baja viscosidad durante el calentamiento, que permite que llene los huecos existentes entre los cristales de óxido de hierro y hace que estén presentes como partículas encerradas por una matriz, en vez de permanecer unidas solamente por una capa delgada de escoria. Sin embargo, a baja temperatura, $\left(1.250{ }^{\circ} \mathrm{C}\right)$ se observa una disminución de la proporción de esco-
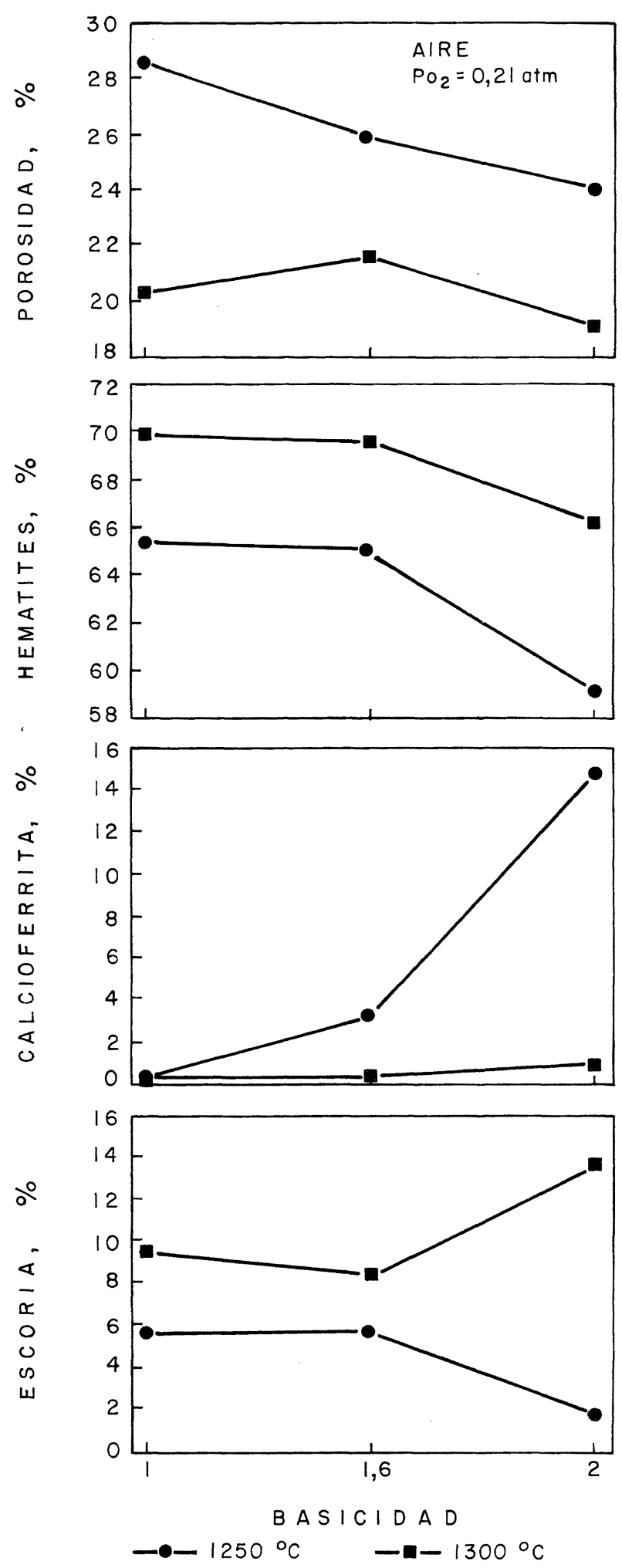

FIG. 4.- Efecto de la temperatura y de la basicidad sobre las proporciones de las fases minerales en briquetas sinterizadas en aire.

FIG. 4.- Effect of temperature and basicity on proportions of mineral phases in briquettes fired in air.

ria cuando el índice de basicidad aumenta, lo que se debe a que parte del $\mathrm{CaO}$ adicionado se consume en 




FIG. 5.- Micrografías de briquetas sinterizadas en aire a $1.250{ }^{\circ} \mathrm{C}$. a) $\mathrm{CaO} / \mathrm{SiO}_{2}=1$, b) $\mathrm{CaO} / \mathrm{SiO}_{2}=2$. $\mathrm{H}$ : hematites, CF: calcioferrita, S: escoria, P: poro.

FIG. 5.- Photomicrographs of briquettes fired in air at 1,250 ${ }^{\circ} \mathrm{C}$. a) $\mathrm{CaO} / \mathrm{SiO}_{2}=1$, b) $\mathrm{CaO} / \mathrm{SiO}_{2}=2$. $H$ : hematite, $C F$ : calcium ferrite, S: slag, P: pore.

la reacción para la formación de calcioferrita y otra parte en la formación de escoria.

De las figuras 4 y 5 se deduce que en las briquetas sinterizadas en aire existe mayor cantidad de calcioferrita y menor cantidad de hematites cuanto menor es la temperatura $\left(1.250{ }^{\circ} \mathrm{C}\right)$. Durante la etapa de calentamiento, la calcioferrita se genera a partir de la reacción de la hematites con el fundente a temperaturas de alrededor de $950-1.100^{\circ} \mathrm{C}$. Cuando se forma la calcioferrita, es estable en una atmósfera de elevado potencial de oxígeno y baja temperatura. Si el medio ambiente cambia a un bajo potencial de oxígeno y alta temperatura, la calcioferrita se transforma a magnetita y silicato líquido. Si la calcioferrita está en un medio ambiente de elevado potencial de oxígeno (aire) y a una temperatura de alrededor de $1.250{ }^{\circ} \mathrm{C}$, se transformará en hematites y silicato líquido. Este fenómeno fue observado y propuesto por otros autores (9). Debido a la transformación de la calcioferrita a hematites y sili- cato líquido, se observa también un incremento de la proporción de escoria a alta temperatura.

\subsubsection{Briquetas sinterizadas en la mezcla gaseosa $0,5 \% \mathrm{O}_{2}-99,5 \% \mathrm{~N}_{2}$}

La influencia de la basicidad a diferentes temperaturas sobre la proporción de fases minerales se muestra en la figura 6 , y se pueden observar sus respectivas micrografías en la figura 7 . De las figuras 6 y 7 , se deduce que la magnetita se incrementa y la hematites disminuye con el aumento de la basicidad. Lo anterior puede explicarse por el cambio de fase que ocurre de hematites a magnetita, el cual tiene lugar a través de la disociación térmica de la hematites.

El equilibrio de la reacción (12):

$$
2 \mathrm{Fe}_{3} \mathrm{O}_{4}+1 / 2 \mathrm{O}_{2} \leftrightarrow 3 \mathrm{Fe}_{2} \mathrm{O}_{3}
$$

se mantiene a la derecha hasta una temperatura de alrededor de $1.400{ }^{\circ} \mathrm{C}$. Sin embargo, a mayor temperatura, comienza una disociación de hematites. El equilibrio cambia a la izquierda causando una vez más la formación de $\mathrm{Fe}_{3} \mathrm{O}_{4}$ y, por lo tanto, un deterioro de la calidad del pelet. Esta disociación de la hematites comienza a más bajas temperaturas si los pelets contienen aditivos básicos. De acuerdo con la cantidad de estos aditivos, la temperatura de disociación disminuye y la porción de magnetita en los pelets se eleva. Este fenómeno se explica por una elevación de temperatura dentro del pelet, debida al calor de formación exotérmico de la calcioferrita y a su calor de fusión adicional, lo cual provoca zonas de sobrecalentamiento en el pelet a temperaturas que exceden $1.400{ }^{\circ} \mathrm{C}$, causando la disociación de la hematites.

Lo anterior ha sido corroborado por otros investigadores (14), que señalan que el contenido de iones ferrosos de los pelets piroconsolidados de magnetita oxidada a temperaturas inferiores a las predichas termodinámicamente, resulta de una temprana disociación de hematites, inducida por los aditivos en los pelets y por los componentes minerales, y no puede atribuirse solamente a la oxidación deficiente de magnetita a hematites. Indican, además, que el contenido de $\mathrm{Fe}^{+2}$ en los aglomerados aumenta con el incremento de la temperatura del proceso de piroconsolidación y con la basicidad.

En adición, la exotermicidad de la oxidación de la magnetita genera una diferencia de temperatura entre el núcleo y la superficie del pelet que puede justificar la disociación de la hematites del núcleo cuando el endurecimiento se realiza a temperaturas próximas a $1.350{ }^{\circ} \mathrm{C}$. A esta temperatura comienza la inversión de la reacción de oxidación donde la 

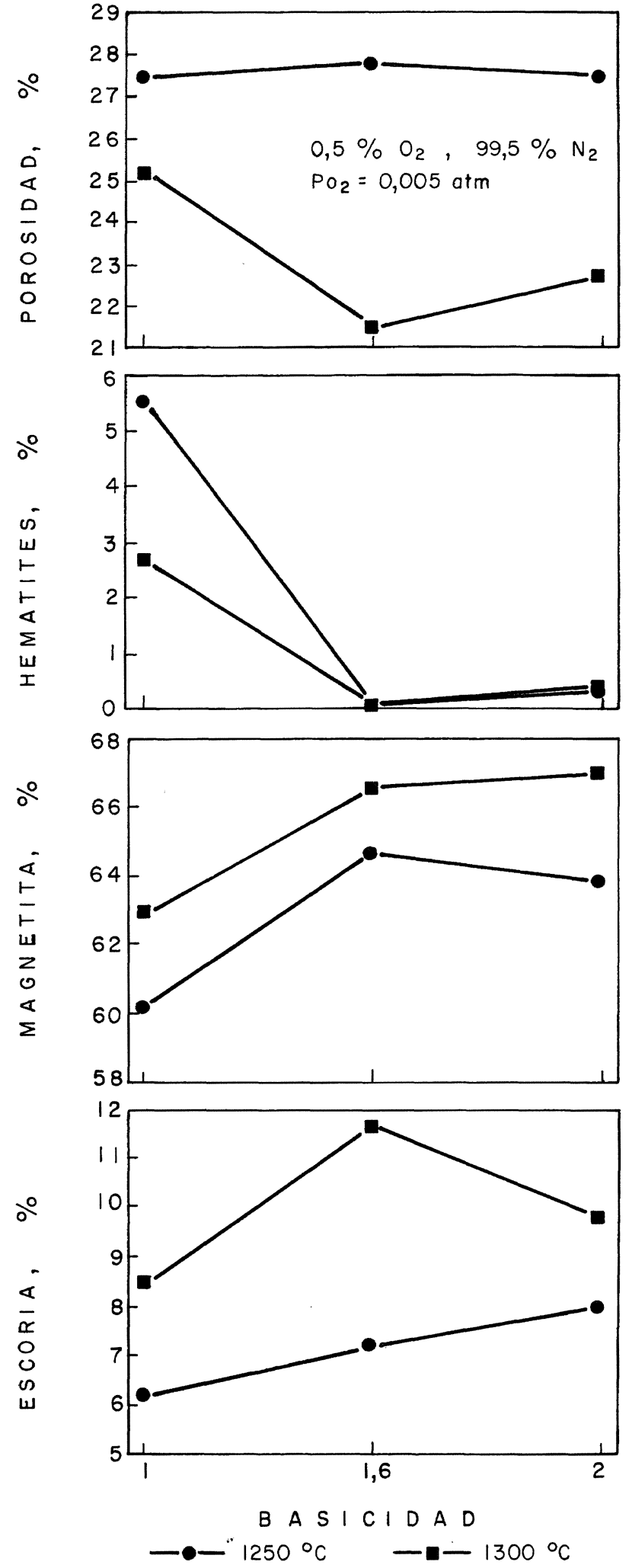

FIG. 6.- Efecto de la temperatura y de la basicidad sobre las proporciones de las fases minerales en briquetas sinterizadas en una mezcla gaseosa de $99,5 \% \mathrm{~N}_{2}$ y $0,5 \% \mathrm{O}_{2}$.

FIG. 6.- Effect of temperature and basicity on proportions of mineral phases in briquettes fired in a gas mixture of $99.5 \% \mathrm{~N}_{2}$ and $0.5 \% \mathrm{O}_{2}$.

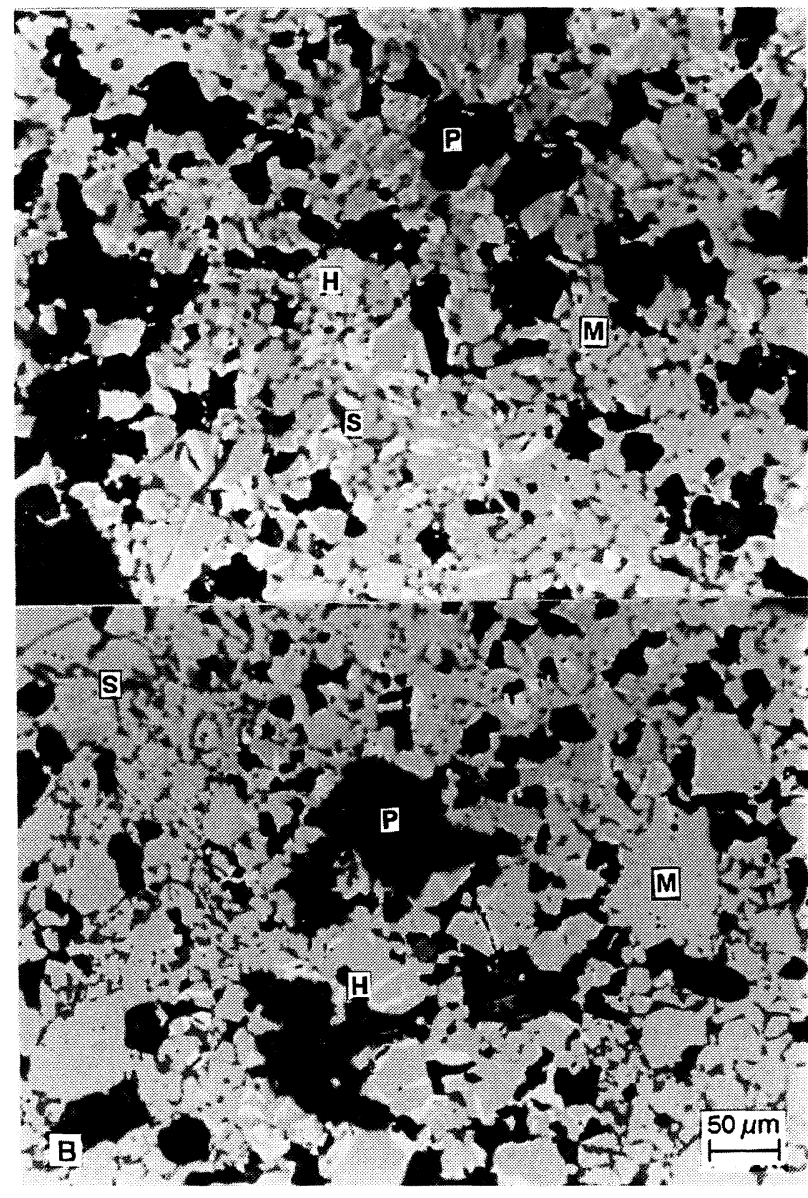

FIG. 7.- Micrografías de briquetas sinterizadas en una mezcla gaseosa de $99,5 \% \mathrm{~N}_{2}-0,5 \% \mathrm{O}_{2}$ a 1.250 ${ }^{\circ} \mathrm{C}$. a) $\mathrm{CaO} / \mathrm{SiO}_{2}=1$, b) $\mathrm{CaO} / \mathrm{SiO}_{2}=2$. H: hematites, M: magnetita, S: escoria, P: poro.

FIG. 7.- Photomicrographs of briquettes fired in a gas mixture of $99.5 \% \mathrm{~N}_{2}-0,5 \% \mathrm{O}_{2}$ at $1,250{ }^{\circ} \mathrm{C}$. a) $\mathrm{CaO} / \mathrm{SiO}_{2}=1$, b) $\mathrm{CaO} / \mathrm{SiO}_{2}=2$. H: hematite, M: magnetite, $S$ : slag, $P$ : pore.

magnetita es más estable, reduciendo la calidad metalúrgica de los pelets (15).

Para este potencial, las tendencias en las proporciones de porosidad y escoria con respecto a la basicidad son similares a las observadas en las muestras sinterizadas en aire, es decir, la disminución de porosidad se acompaña de un incremento de la cantidad de escoria cuando la basicidad aumenta para las dos temperaturas analizadas $\left(1.250\right.$ y $\left.1.300{ }^{\circ} \mathrm{C}\right)$. En este caso, el incremento de la cantidad de escoria con el contenido de $\mathrm{CaO}$ a $1.250{ }^{\circ} \mathrm{C}$ se debe a que el $\mathrm{CaO}$ reacciona con los óxidos de hierro para producir solamente pequeñas cantidades de calcioferrita, y la mayor parte del $\mathrm{CaO}$ constituye la escoria.

De las figuras 6 y 7 se puede deducir que a menor temperatura corresponde una mayor cantidad de hematites y menor cantidad de magnetita. Aquí, la velocidad de oxidación parece estar relacionada 
con el desarrollo del silicato líquido y con las estabilidades relativas de los óxidos de hierro, por ejemplo, la disociación térmica de la hematites. A bajas temperaturas, cuando el líquido se desarrolla más lentamente, la oxidación es relativamente rápida. A altas temperaturas (superiores a $1.250^{\circ} \mathrm{C}$ ), el silicato líquido se forma rápidamente y esto perjudica la oxidación gaseosa de la magnetita y, por lo tanto, disminuye la velocidad global de oxidación.

Existen también cambios en la porosidad, que disminuye con el incremento de la temperatura, ya que ésta favorece el crecimiento de grano de los óxidos de hierro reduciendo el tamaño de los poros y cambiando su forma irregular a esférica. Para los tres potenciales estudiados se encontró una tendencia similar.

\subsubsection{Briquetas sinterizadas en la mezcla gaseosa $1 \% \mathrm{CO}-24 \% \mathrm{CO}_{2}-75 \% \mathrm{~N}_{2}$}

Como la etapa de calentamiento se llevó a cabo en una atmósfera con bajo potencial de oxígeno, no fueron posibles las reacciones de oxidación de magnetita a hematites y la reacción de hematites y óxido de calcio para formar calcioferrita; por lo tanto, las reacciones de interés para la formación de las fases minerales finales se produjeron durante el enfriamiento en aire.

Las figuras 8 y 9 muestran la influencia de la basicidad y de la temperatura en la proporción de las fases minerales. Se encontró que a la temperatura máxima de $1.250^{\circ} \mathrm{C}\left(\mathrm{pO}_{2}=2,86 \times 10^{-8} \mathrm{~atm}\right.$. $)$, las principales fases minerales presentes eran hematites, calcioferrita, escoria y poros. Durante el estado de enfriamiento, cerca de los poros, la magnetita remanente después de la etapa de calentamiento se enfría en una atmósfera de elevado potencial de oxígeno $(0,21 \mathrm{~atm}$.) y tiende a oxidarse a hematites. En las regiones distantes de los poros, que se enfrían en un potencial de oxígeno menor de 0,21 atm., la magnetita presente reacciona con el silicato líquido y el oxígeno para producir calcioferrita.

Se puede observar también que la cantidad de calcioferrita se eleva con el incremento del índice de basicidad, a la vez que disminuye la proporción de escoria. En este caso, el óxido de calcio presente en el aglomerado no reacciona con la magnetita durante la etapa de calentamiento por encontrarse en una atmósfera muy reductora, y únicamente forma silicatos que reaccionan con la magnetita y con el oxígeno durante el enfriamiento a elevado potencial oxidante para formar la calcioferrita. Cuanto mayor sea la cantidad de óxido de calcio, mayor será la cantidad de silicato formado que tenderá a reaccionar con la magnetita, aumentando así la cantidad de calcioferrita y disminuyendo la proporción de escoria final.
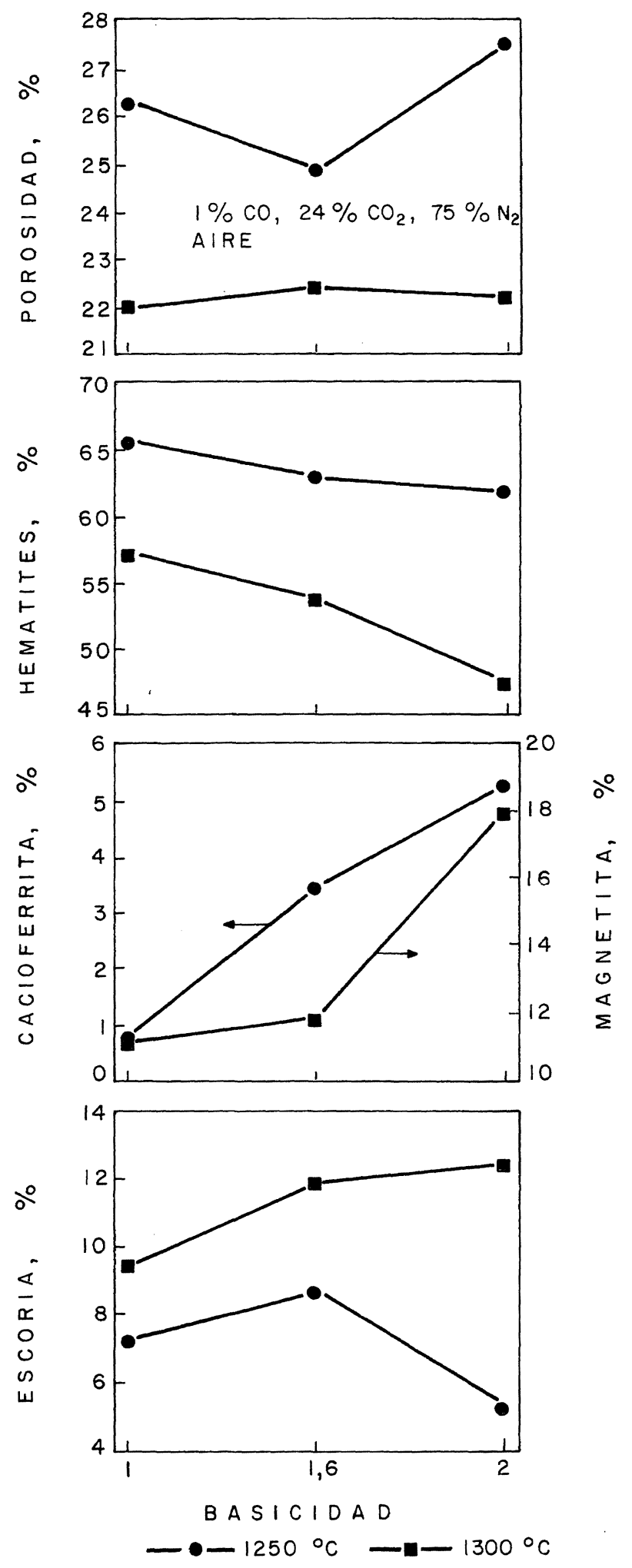

FIG. 8. - Efecto de la temperatura y de la basicidad sobre las proporciones de las fases minerales en briquetas sinterizadas en una mezcla gaseosa de $1 \%$ $\mathrm{CO}-24 \% \mathrm{CO}_{2}-75 \% \mathrm{~N}_{2}$-aire.

FIG. 8.- Effect of temperature and basicity on proportions of mineral phases in briquettes fired in a gas mixture of $1 \% \mathrm{CO}-24 \% \mathrm{CO}_{2}-75 \% \mathrm{~N}_{2}$-air. 


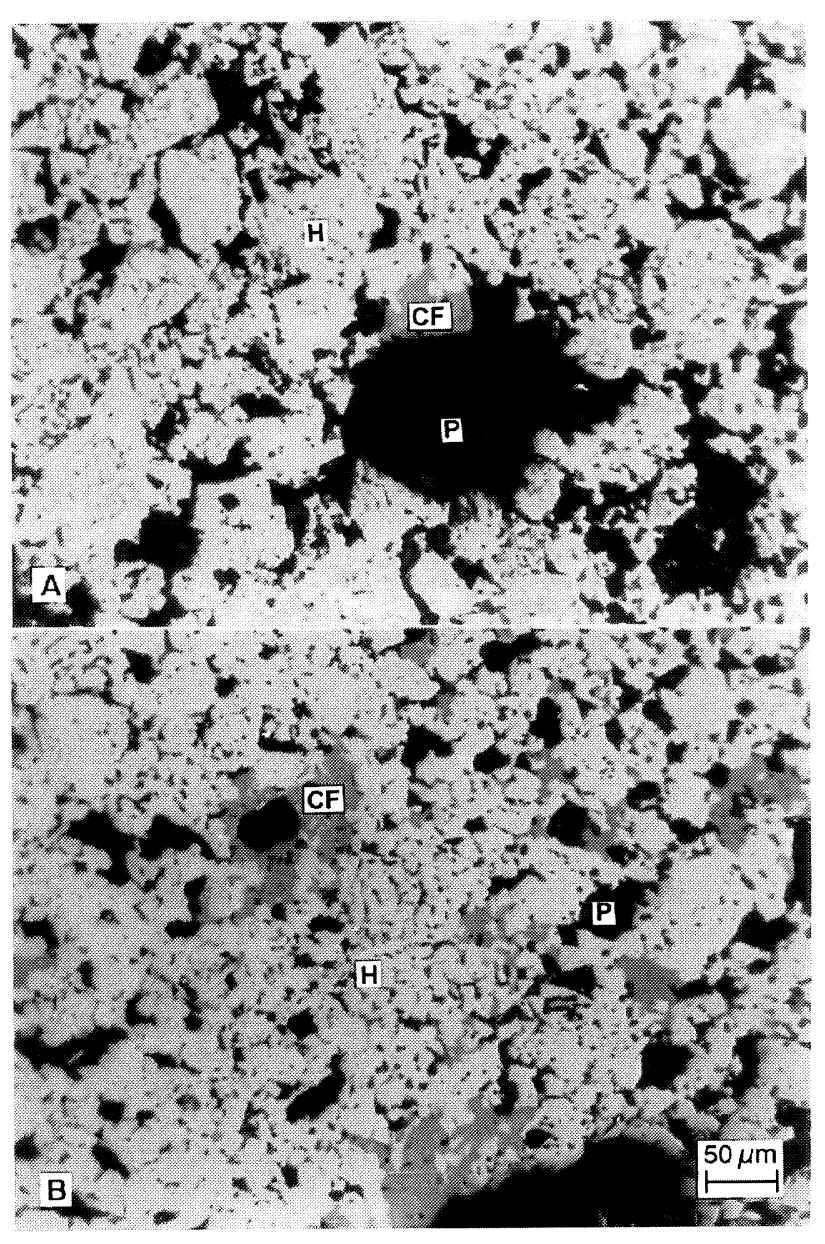

FIG. 9.- Micrografías de briquetas sinterizadas en una mezcla gaseosa de $1 \% \mathrm{CO}-24 \% \mathrm{CO}_{2}-75 \% \mathrm{~N}_{2}$ a $1.250{ }^{\circ} \mathrm{C}$. a) $\mathrm{CaO} / \mathrm{SiO}_{2}=1$, b) $\mathrm{CaO} / \mathrm{SiO}_{2}=2 . \mathrm{H}$ : hematites, M: magnetita, CF: calcioferrita, S: escoria, P: poro.

FIG. 9.- Photomicrographs of briquettes fired in a gas mixture of $1 \%$ CO-24 \% CO $\mathrm{CO}_{2}-75 \% \mathrm{~N}_{2}$ at $1,250{ }^{\circ} \mathrm{C}$. a) $\mathrm{CaO} / \mathrm{SiO}_{2}=1$, b) $\mathrm{CaO} / \mathrm{SiO}_{2}=2$. $H$ : hematite, $M$ : magnetite, $C F$ : calcium ferrite, S: slag, P: pore.

Ahsan y col. (16) concluyeron que la calcioferrita tiene un amplio intervalo de composición asociado con un tipo de estructura cristalina, una superred de magnetita. Consideraron que se forma por una sustitución de átomos de aluminio, silicio y calcio dentro de la red de magnetita, en lugar de por átomos de hierro. En su investigación se sostiene la propuesta de que la magnetita reacciona con diferentes cantidades de $\mathrm{CaO}$ y $\mathrm{SiO}_{2}$ (del líquido) para formar la calcioferrita con un amplio rango de composición. De esta manera, la composición de esta ferrita está probablemente relacionada tanto con las cantidades de los constituyentes $\left(\mathrm{CaO}, \mathrm{SiO}_{2}\right.$ y $\mathrm{Al}_{2} \mathrm{O}_{3}$ ) que rodean la magnetita como con la temperatura de reacción.

A $1.300{ }^{\circ} \mathrm{C}\left(\mathrm{pO}_{2}=1,18 \times 10^{-7} \mathrm{~atm}\right.$.), se observa que las fases predominantes en los aglomerados fue- ron magnetita, hematites, escoria y poros, observándose la ausencia de calcioferrita. Las figuras 8 y 9 muestran que, con respecto al índice de basicidad, las proporciones de las fases de magnetita y de hematites presentaron comportamientos similares a los aglomerados sinterizados en atmósfera de $0,5 \% \mathrm{O}_{2}-99,5 \%$ $\mathrm{N}_{2}$, es decir, la proporción de magnetita se incrementa y la de hematites disminuye con el aumento del índice de basicidad. Lo anterior se puede explicar por el cambio de fase que ocurre de hematites a magnetita, que tiene lugar a través de la disociación térmica de la hematites mencionada anteriormente.

\subsection{Efecto de la velocidad de enfriamiento en la formación de fases minerales}

Con respecto a la influencia de la velocidad de enfriamiento en la proporción de las fases minerales en briquetas sinterizadas a diferentes temperaturas, se puede observar que la cantidad de calcioferrita y hematites tiende a disminuir cuando la velocidad de enfriamiento aumenta, con los mayores niveles de calcioferrita y menores niveles de hematites asociada con la menor temperatura. Se sabe que un rápido enfriamiento, desde la máxima temperatura de sinterización, es perjudicial para el crecimiento de grano y para la recristalización del líquido de los óxidos de hierro, pero una vez finalizada la solidificación del silicato líquido, la velocidad de enfriamiento no tiene mucho efecto sobre las fases minerales.

Los resultados para las proporciones de las fases minerales, en función de la velocidad de enfriamiento para briquetas sinterizadas en el oxidante potencial de $5 \times 10^{-3} \mathrm{~atm}$., indican que un incremento de magnetita acompaña a una disminución de la cantidad de hematites cuando la velocidad de enfriamiento aumenta para las dos temperaturas estudiadas. Se sabe que una velocidad lenta de enfriamiento favorece la oxidación de magnetita a hematites en una elevado potencial de oxígeno, mientras que un rápido proceso de enfriamiento puede conservar los minerales formados en la etapa de calentamiento y retardar la formación de calcioferrita o la oxidación de magnetita a hematites.

Para las muestras sinterizadas en el tercer potencial oxidante $\left(\mathrm{pO}_{2}=1,18 \times 10^{-7} \mathrm{~atm}\right.$. ) a $1.300^{\circ} \mathrm{C}$, las tendencias en la proporción de hematites y magnetita son similares a las observadas en las muestras sinterizadas a $5 \times 10^{-3} \mathrm{~atm}$., pero en este caso, los cambios globales de los porcentajes de las fases son mayores. La hematites disminuye de 64 a $42 \%$ y la magnetita aumenta de 2,5 a $25 \%$, aproximadamente. Para $1.250{ }^{\circ} \mathrm{C}\left(\mathrm{pO}_{2}=2,86 \times 10^{-8} \mathrm{~atm}\right.$.), la cantidad de hematites se mantuvo relativamente constante en el $64 \%$ y la calcioferrita presentó un ligero aumento con la velocidad de enfriamiento. 
El comportamiento de las tendencias de las cantilades de escoria y de la porosidad es completamente somplejo. Para las briquetas sinterizadas al aire, se suede observar un ligero aumento de la proporción de estas fases cuando la velocidad de enfriamiento lumenta, con una cantidad mayor de escoria y porosidad menor a mayor temperatura. Para las muestras sinterizadas en el potencial oxidante de $5 \times 10^{-3}$ atm., a $1.250{ }^{\circ} \mathrm{C}$ se observa un aumento de la porosidad acompañado de una disminución de la cantilad de escoria cuando la velocidad de enfriamiento uumenta y, por el contrario, a $1.300{ }^{\circ} \mathrm{C}$, la porosidad sarece disminuir y la cantidad de escoria tiende a zumentar con la velocidad de enfriamiento. Finalmente, para la mezcla gaseosa $1 \% \mathrm{CO}-24 \% \mathrm{CO}_{2}-$ $75 \% \mathrm{~N}_{2}$, se puede observar una disminución de la porosidad con el aumento de la velocidad de enfriamiento, mientras la escoria parece que se mantiene ən un valor constante del $11 \%$.

\section{CONCLUSIONES}

- Se comprobó, por medio de la experimentación, que la variación de las condiciones de sinterización, tales como basicidad, temperatura, ciclo térmico y potencial oxidante, influyen acusadamente en el tipo y proporción de las fases minerales presentes y en la estructura final del aglomerado.

- A mayor potencial oxidante, se presenta mayor cantidad de hematites, producto de la oxidación de la magnetita durante el estado de calentamiento.

- La calcioferrita está presente a elevado potencial oxidante debido a su formación a partir de la hematites secundaria originada por la oxidación de la magnetita.

- La calcioferrita se origina también por la reacción de la magnetita, silicato líquido y oxígeno durante el enfriamiento a elevado potencial oxidante.

- El aumento del índice de basicidad favorece la reacción del $\mathrm{CaO}$ con la hematites para la formación de calcioferrita, para briquetas sinterizadas en aire.

- El incremento de la basicidad provoca la disociación térmica de hematites a magnetita a bajos potenciales oxidantes, cuando se trabaja con elevadas temperaturas de sinterización.
- La porosidad de las probetas sinterizadas en los tres potenciales oxidantes estudiados disminuye con el aumento de la basicidad debido a la formación de escoria fluida durante la etapa de calentamiento.

- La inestabilidad de la calcioferrita a altas temperaturas origina un aumento de la proporción de hematites y escoria.

\section{Agradecimiento}

Los autores agradecen al CONACYT y a la Universidad Michoacana de San Nicolás de Hidalgo el apoyo brindado para la realización de este trabajo.

\section{REFERENCIAS}

(1) Fitton, J.T. y GoldRing, D.C. J. Iron Steel Inst. London, May. 1966: 452-459.

(2) Ball, D.F., Fitton, J.T., Dawson, P.R. y Goldring, D.C. Miner. Process. Extractive Metall., 83, 1974: C47C58.

(3) Panigrahy, S.C., Verstraeten, P. y Dilewijns, J. Ironmaking Steelmaking, 11 (1), 1984: 17-22.

(4) Bedolla, B. Egberto, Mendoza, G., S. Guillermo y LEMUS, J. ISIJ Int., 34 (8), 1994: 631-640.

(5) Bedolla E., Leon C.A., Lemus J., Aguilar, E.A. y MenDOZA G., Rev. Metal. Madrid, 31 (6), 1995: 368-378.

(6) John, J., Friel y Edwin S. ERICKSON, Jr. Metall. Trans. B, 11B (Jun.), 1980: 233-243.

(7) Friel, J.J. y ERICKSON, Jr., E.S. Soc. Min. Eng. AIME, 272, 1980: 1.843-1.845.

(8) Hsieh, Li-Heng y Whiteman, J.A. ISIJ Int., 29 (8), 1989: 625-630.

(9) Hsien, Li-Heng y Whiteman, J.A. ISIJ Int., 29 (1), 1989: 24-32.

(10) Hsieh, Li-Heng y Whiteman, J.A. ISIJ Int., 33 (4), 1993: 462-473.

(11) ERvin, E. Underwood, Quantitative Metallography. Metals Handbook. Vol. 10. Materials Characterization. Metallographic Techniques. $9^{a}$ ed. American Society for Metals. Metals Park, Ohio 44073. 1986: 123.

(12) Meyer, K. Pelletizing of Iron Ores. Springer-Verlag. Berlín- Heidelberg-Nueva York, 1980: 99.

(13) Wright, J. Powder Technol., 30, 1981: 185-194.

(14) Ferreira, S., Siguin, D. y Garcia, F. Ironmaking Steelmaking, 21 (3), 1994: 244-246.

(15) Ruiz Sierra, J.C., Badie, J.M. y Chejne Janna, F. Ironmaking Steelmaking, 21 (2), 1994: 114-117.

(16) Ahsan, S.N., Mukherjee, T. y Whiteman, J.A. Ironmaking Steelmaking, 10 (2), 1983: 54-64. 